\title{
NÚMERO MÍNIMO DE TENSÕES PARA DETERMINAÇÃO DA CURVA CARACTERÍSTICA DE RETENÇÃO DE ÁGUA DE UM LATOSSOLO VERMELHO EUTRÓFICO SOB SISTEMA DE SEMEADURA DIRETA ${ }^{(1)}$
}

\author{
Ricardo Falqueto Jorge ${ }^{(2)}$, José Eduardo Corá ${ }^{(3)}$ \& José Carlos Barbosa ${ }^{(4)}$
}

\begin{abstract}
RESUMO
Para o conhecimento do comportamento físico-hídrico do solo, é fundamental a determinação da curva de retenção de água (CRA). A aquisição de dados para obtenção da CRA envolve processos demorados e custos elevados. A hipótese deste estudo foi a de que é possível determinar a CRA com menor número de tensões, reduzindo seu tempo de obtenção, sem, contudo, comprometer a acurácia dos resultados. Assim, o objetivo deste trabalho foi determinar quais tensões podem ser utilizadas para determinação da CRA de Latossolo Vermelho eutrófico de maneira acurada e em menor tempo. Foram determinados os conteúdos de água retidos em amostras de um Latossolo Vermelho eutrófico sob sistema de semeadura direta (SSD). As tensões utilizadas na determinação dos conteúdos de água foram: $0,10,20,40,60,80,100,200,300,500,700,1.000,3.000,5.000$ e $15.000 \mathrm{hPa}$. Os dados foram combinados e determinaram-se 450 CRAs ajustadas pelo modelo de van Genuchten. Os parâmetros $\alpha, \mathrm{m}, \mathrm{n}$ e $\boldsymbol{U g}_{\text {res }}$, gerados pelo modelo, foram submetidos à análise de variância (teste F) e as médias comparadas pelo teste de Scott-Knott $(p=0,05)$. A hipótese deste estudo foi confirmada, ou seja, é possível determinar a CRA com menor número de tensões, reduzindo seu tempo de obtenção em até cinco vezes, sem, contudo, comprometer a acurácia dos resultados. A tensão de $15.000 \mathrm{hPa}$ deve estar contida na combinação de tensões a serem utilizadas para determinação da curva característica de retenção de água, quando se emprega o modelo matemático de van Genuchten para ajuste dos dados. As curvas características de retenção de água do solo, definidas com as combinações de tensões $(0,60,700$ e $15.000 \mathrm{hPa}),(0,80,700$ e $15.000 \mathrm{hPa}),(0,100,1.000$ e $15.000 \mathrm{hPa})$, $(0,20,60,100,700$ e $15.000 \mathrm{hPa}),(0,80,300,700$ e $15.000 \mathrm{hPa})$ e $(0,100,300,1.000$ e $15.000 \mathrm{hPa}$ ), sendo os dados ajustados ao modelo de van Genuchten, proporcionaram
\end{abstract}

\footnotetext{
(1) Retirado da Tese de Doutorado do primeiro autor. Recebido para publicação em dezembro de 2009 e aprovado em outubro de 2010.

${ }^{(2)}$ Doutorando do Programa de Pós-graduação em Agronomia (Produção Vegetal), Universidade Estadual Paulista - UNESP. Campus de Jaboticabal. Via de Acesso Professor Paulo Donato Castellane s/n, CEP 14884-900 Jaboticabal (SP). Bolsista CAPES. E-mail: jrfalqueto@yahoo.com.br

(3) Professor Adjunto do Departamento de Solos e Adubos, UNESP. Campus de Jaboticabal. Bolsista CNPq. E-mail: cora@fcav.unesp.br

(4) Professor Titular do Departamento de Ciências Exatas, UNESP. Campus de Jaboticabal. Bolsista CNPq. E-mail: jcbarbosa@fcav.unesp.br
} 
estimativas dos conteúdos de água, retidos no Latossolo Vermelho eutrófico sob sistema de semeadura direta, com a mesma acurácia, quando comparada à curva de referência partindo-se de um conjunto de 15 valores de tensões.

Termos de indexação: modelo de van Genuchten, atributos físicos do solo, física do solo.

\title{
SUMMARY: MINIMUM NUMBER OF TENSION MEASUREMENTS TO DETERMINE THE SOIL-WATER RETENTION CURVE OF A EUTROPHIC RED OXISOIL UNDER NO-TILLAGE
}

\begin{abstract}
The soil-water retention curve (SWRC) is essential to understand the physical-hydric soil behavior. However, data for the construction of the SWRC are obtained in slow and expensive processes. This study hypothesized the possibility that SWRC could be constructed based on fewer tension data within a shorter time, without affecting the accuracy. The objective was to determine the tension data required for the construction of an accurate SWRC in less time. Undisturbed soil samples were collected from a Eutrophic Red Oxisol under long-term notillage. For the SWRC construction, soil water contents were evaluated at tensions of 0; 10; 20; 40; 60; 80; 100; 200; 300; 500; 700; 1,000; 3,000; 5,000, and 15,000 hPa. Combining the 15 tensions used, 450 SWRC were obtained and adjusted by the van Genuchten model. Variance analysis for the $\alpha, m, n$ and $\mathrm{Ug}_{\text {res }}$ parameters generated for the model were considered for statistical procedures. Means were subjected to the Scott-Knott cluster analysis procedure $(p=0.05)$. The hypothesis that the SWRC can be constructed with fewer tension measurements within a shorter time, without reducing the accuracy, was confirmed. The 15,000 hPa tension must be included in the combination of tensions used to determine the SWRC, if the adjusted van Genuchten model is applied for data fitting. The Eutrophic Red Oxisol water retention curve determined with the combined data at tensions of 0; 60; 700; 15,000 hPa, 0; 80; 700; 15,000 hPa, 0; 100; 1,000; 15,000 hPa, 0; 20; 60; 100; 700; 15,000 hPa, 0; 80; 300; 700; 15,000 hPa and 0; 100; 300; 1,000; 15,000 hPa by the van Genuchten adjusted model estimated the soil water content at different tensions with the same accuracy, compared to the reference curve based on a set of 15 tension measurements.
\end{abstract}

Index terms: van Genuchten model, soil physical properties, soil physics.

\section{INTRODUÇÃO}

A capacidade de um solo reter e disponibilizar água para as plantas é representada pela sua curva de retenção de água (CRA), que é a relação entre o conteúdo de água no solo e a tensão na qual ela está retida. Dessa forma, a CRA é elaborada pela determinação do conteúdo de água no solo em diferentes tensões.

Normalmente, as tensões utilizadas para determinação da CRA são selecionadas de forma arbitrária (Silva et al., 2006). Assim, a variabilidade metodológica usada para medir a CRA é grande. De acordo com Silva et al. (2006), geralmente são usados de seis a oito pontos de tensão na determinação da CRA, dependendo do modelo matemático adotado. Como não há uma padronização dos valores de tensão a serem utilizados, Silva et al. (2006) sugerem que a CRA deve ser determinada com o maior número de tensões possíveis. Dexter et al. (2007) e Machado et al. (2008), em trabalhos que se utilizaram da CRA, a elaboraram utilizando 11 tensões, enquanto Blainski et al. (2008) e Silva et al. (2008) utilizaram 12 para sua determinação.

$\mathrm{Na}$ literatura são encontrados vários modelos matemáticos para o ajuste da CRA (Dourado-Neto et al., 2000). O modelo proposto por van Genuchten (1980) é, provavelmente, o mais utilizado para predizer os parâmetros que descrevem a CRA (Dexter et al., 2008; Tormena et al., 2008). Matematicamente, quanto maior o número de tensões utilizadas na determinação do conteúdo de água, melhor será o ajuste do modelo aos dados e, consequentemente, a acurácia e representatividade da CRA.

Entretanto, o uso de maior número de tensões para determinar a CRA torna o método lento e oneroso (Hodnett \& Tomasella, 2002; Silva et al., 2006). Adicionalmente, com a utilização de grande número de tensões, aumenta-se o risco de alteração da estrutura do solo das amostras, devido à maior frequência de manipulação delas, o que acarretará baixa qualidade dos dados (Moraes et al., 1993). 
O tempo para a determinação da CRA também aumenta em decorrência das tensões utilizadas. Para o método que emprega a câmara de pressão de Richards na determinação da CRA, o qual tem sido um dos mais utilizados (Machado et al., 2008; Silva et al., 2008; Tormena et al., 2008), as amostras de solo atingem o equilíbrio físico-hídrico em menor tempo quando submetidas às menores tensões $(\leq 100 \mathrm{hPa})$, em relação às maiores tensões $(>100 \mathrm{hPa})$. Desse modo, para determinação da CRA em menor tempo, deve-se priorizar, na medida do possível, um número maior de tensões de menores valores $(\leq 100 \mathrm{hPa})$.

A utilização arbitrária de um determinado conjunto de tensões, sem um critério de seleção definido, pode resultar em uma estimativa da CRA não representativa do solo. Por isso, é fundamental a definição de um número mínimo de tensões que resulte na determinação acurada da CRA, em menor intervalo de tempo. Nesse sentido, a hipótese deste estudo foi a de que é possível determinar a CRA com menor número de tensões, reduzindo seu tempo de obtenção, sem, contudo, comprometer a acurácia dos resultados. Assim, o objetivo deste trabalho foi determinar quais tensões podem ser usadas na determinação da curva de retenção de água de um Latossolo Vermelho eutrófico de maneira acurada e visando menor tempo.

\section{MATERIAL E MÉTODOS}

O estudo foi realizado em uma área experimental de dois hectares, conduzida sob sistema de semeadura direta (SSD) desde 2002, no município de JaboticabalSP $\left(21^{\circ} 15^{\prime} 22^{\prime \prime} \mathrm{S}\right.$ e $48^{\circ} 18^{\prime} 58^{\prime} \mathrm{W}$ e $550 \mathrm{~m}$ de altitude média). O solo foi classificado como um Latossolo Vermelho eutrófico típico, de acordo com os critérios do Sistema Brasileiro de Classificação de Solos (Embrapa, 2006).

Para elaboração da curva característica de retenção de água do solo, em outubro de 2006 , foram coletadas amostras com estrutura do solo indeformada, em parcelas de um tratamento de longa duração (iniciado em 2002), conduzido na área experimental. O tratamento consiste do cultivo de soja em monocultura como cultura de verão e, na entressafra (cultivo de outono/inverno), do cultivo de sete diferentes culturas: milho, girassol, nabo forrageiro, milheto, guandu, sorgo e crotalária, nas mesmas parcelas todos os anos. $\mathrm{O}$ experimento foi constituído de três repetições, totalizando, portanto, 21 parcelas (sete culturas de inverno e três repetições). Foram coletadas três amostras (replicatas) em cada parcela, nas camadas de $0,0-0,1,0,1-0,2$ e $0,2-0,3 \mathrm{~m}$ do solo, utilizando-se anéis volumétricos de $0,05 \mathrm{~m}$ de diâmetro e $0,05 \mathrm{~m}$ de altura, perfazendo um total de 189 amostras (21 parcelas $\mathrm{x}$ três profundidades $\mathrm{x}$ três replicações). Nas mesmas parcelas e camadas foram coletadas amostras deformadas de solo (total de 189 amostras) para caracterização granulométrica, seguindo método proposto pela Embrapa (1997) (Quadro 1).

As amostras com estrutura indeformada foram colocadas para serem saturadas por água durante $24 \mathrm{~h}$, por meio da elevação gradual de uma lâmina de água, até atingirem cerca de dois terços da altura do anel. Em seguida, cada amostra foi submetida progressivamente às tensões: $0,10,20,40,60,80 \mathrm{e}$ $100 \mathrm{hPa}$, utilizando-se mesa de tensão, e 200, 300, $500,700,1.000,3.000,5.000$ e $15.000 \mathrm{hPa}$, utilizandose câmara de pressão de Richards com placa porosa (Klute, 1986). Visando menor tempo para as amostras atingirem o equilíbrio hídrico, a retenção de água nas tensões de $3.000,5.000$ e $15.000 \mathrm{hPa}$ foram determinadas em amostras deformadas de solo (porção de terra fina seca ao ar, passada em peneira de $2 \mathrm{~mm}$ ), acondicionadas em anel de borracha ou de PVC com $0,01 \mathrm{~m}$ de altura, colocadas diretamente sobre a placa porosa (Embrapa, 1997).

Ao atingir o equilíbrio hídrico em cada tensão, cada amostra foi pesada e, após submetidas à última tensão, foram secas em estufa a $105{ }^{\circ} \mathrm{C}$ durante $24 \mathrm{~h}$, para determinação do conteúdo de água retido em cada tensão (Gardner, 1986). Foram obtidos 63 valores (médias das três replicatas) do conteúdo de água retido nas amostras (Quadro 2).

Utilizando o conjunto de dados, correspondentes aos conteúdos de água retidos nas 189 amostras do solo em cada uma das 15 tensões utilizadas $(0,10,20$, $40,60,80,100,200,300,500,700,1.000,3.000,5.000$ e $15.000 \mathrm{hPa}$ ) foram definidas 450 combinações, levando em conta o número e os valores de tensão, objetivando o ajuste da curva característica de retenção de água no solo (CRA). O valor do conteúdo de água na saturação (tensão $0 \mathrm{hPa}$ ) foi utilizado em todas as 450 combinações propostas. O critério adotado na escolha do conjunto de dados para cada uma das 450 combinações foi o de utilização do maior número possível de tensões de menores valores $(<200 \mathrm{hPa})$, visando menor tempo para construção da curva, pois, quanto maior o valor da tensão aplicada, maior é o

Quadro 1. Valores médios, mínimos e máximos dos teores de areia, silte, argila e de densidade do solo (Ds) das amostras do Latossolo Vermelho eutrófico utilizadas na elaboração das curvas características de retenção de água do solo

\begin{tabular}{lrrrc}
\hline & Areia & Silte & Argila & Ds \\
\cline { 2 - 4 } & & g kg $^{-1}$ & & $\mathrm{Mg} \mathrm{m}^{-3}$ \\
Média geral & 364 & 70 & 566 & 1,31 \\
Mínimo & 199 & 20 & 379 & 1,12 \\
Maximo & 567 & 114 & 720 & 1,65 \\
Desvio-padrão & 102 & 21 & 87 & 0,12 \\
CV (\%) & 28 & 30 & 15 & 9,45 \\
\hline
\end{tabular}


Quadro 2. Valores médios, mínimos e máximos do conteúdo de água $\left(\mathrm{kg} \mathrm{kg}^{-1}\right)$ retido nas amostras utilizadas na elaboração das curvas características de retenção de água do solo, em função das tensões aplicadas

\begin{tabular}{|c|c|c|c|c|c|c|c|c|c|c|c|c|c|c|c|}
\hline & \multicolumn{15}{|c|}{ Tensão (hPa) } \\
\hline & $0,0^{(1)}$ & 10 & 20 & 40 & 60 & 80 & 100 & 200 & 300 & 500 & 700 & 1.000 & 3.000 & 5.000 & 15.000 \\
\hline & \multicolumn{15}{|c|}{ Conteúdo de água no solo $\left(\mathrm{kg} \mathrm{kg}^{-1}\right)$} \\
\hline Média geral & 0,38 & 0,36 & 0,32 & 0,29 & 0,27 & 0,26 & 0,25 & 0,23 & 0,22 & 0,21 & 0,20 & 0,19 & 0,18 & 0,17 & 0,15 \\
\hline Mínimo & 0,23 & 0,22 & 0,22 & 0,21 & 0,20 & 0,19 & 0,18 & 0,17 & 0,16 & 0,15 & 0,14 & 0,13 & 0,12 & 0,11 & 0,10 \\
\hline Máximo & 0,51 & 0,49 & 0,42 & 0,36 & 0,33 & 0,32 & 0,31 & 0,29 & 0,27 & 0,26 & 0,25 & 0,24 & 0,23 & 0,22 & 0,20 \\
\hline Desvio-padrão & 0,07 & 0,06 & 0,05 & 0,04 & 0,04 & 0,04 & 0,04 & 0,04 & 0,03 & 0,03 & 0,03 & 0,03 & 0,03 & 0,03 & 0,03 \\
\hline $\mathrm{CV}(\%)$ & 18 & 17 & 15 & 13 & 13 & 14 & 14 & 15 & 15 & 15 & 16 & 16 & 17 & 18 & 17 \\
\hline
\end{tabular}

tempo necessário para obtenção do equilíbrio hídrico das amostras e maiores são os problemas causados pelo efeito da histerese (Moraes et al., 1993).

A combinação que considerou o conjunto dos dados referentes a todas as 15 tensões foi considerada a de referência para a comparação com as demais combinações e, no presente estudo, foi denominada de curva característica de retenção de água do solo C1. As demais combinações foram definidas com os seguintes números de tensões: nove combinações utilizando-se o conjunto de dados com 14 tensões, as quais foram definidas excluindo-se uma das seguintes tensões da curva de referência (C1): 10, 80, 100, 500, 700, 1.000, 3.000, 5.000 e 15.000 hPa; onze combinações utilizando-se o conjunto de dados com 13 tensões, definidas excluindo-se um dos seguintes pares de tensões de C1: 20 e 500; 300 e 700; 3.000 e 5.000; 1.000 e $3.000 ; 700$ e 1.000; 500 e $1.000 ; 500$ e 700; 10 e 700; 10 e 5.000; 10 e 3.000; ou 1.000 e 15.000 hPa; dezenove combinações referentes ao conjunto de dados com 12 tensões, definidas excluindo-se um dos seguintes conjuntos de três tensões de C1: (10; 500; $5.000),(10 ; 200 ; 500),(10 ; 300 ; 500),(200 ; 500 ; 15.000)$, (300; 500; 15.000), (1.000; 3.000; 15.000), (40; 500; $5.000),(40 ; 200 ; 500),(40 ; 300 ; 500),(100 ; 200 ; 500)$, (100; 300; 500), (100; 300; 700), (200; 500; 700), (500; $1.000 ; 5.000),(1.000 ; 3.000 ; 5.000),(200 ; 500 ; 3.000)$, (200; 500; 5.000), (1.000; 5.000; 15.000), e (3.000; 5.000; $15.000 \mathrm{hPa}$ ). As demais combinações foram definidas excluindo-se tensões da curva $\mathrm{C} 1$ de forma aleatória, visando um número maior possível de tensões de menores valores. Dessa maneira, 24 combinações foram definidas com 11 tensões, 36 combinações com 10 tensões, 51 com nove tensões, 61 com oito tensões, 71 com sete tensões, 64 com seis tensões, 55 com cinco tensões, 43 com quatro tensões e cinco com três tensões, sendo elas $(0 ; 10 ; 15.000),(0 ; 20 ; 15.000),(0$; $60 ; 15.000),(0 ; 80 ; 15.000)$ e $(0 ; 100 ; 15.000) \mathrm{hPa}$, totalizando, portanto, as 450 combinações propostas.

Os dados do conteúdo de água retido em cada tensão $(U g)$ foram ajustados ao modelo de van Genuchten (1980) (Equação 1), para cada uma das 450 combinações:

$$
U g=\left(U g_{s a t}-U g_{r e s}\right) /\left[1+(a h)^{\mathrm{n}}\right]^{\mathrm{m}}+U g_{\text {res }}
$$

em que $\alpha\left(\mathrm{hPa}^{-1}\right), n$ e m são parâmetros da curva de retenção de água no solo; $h$ é a tensão da água no solo (hPa); $U g_{\text {sat }}$ e $U g_{\text {res }}$ são os conteúdos de água na saturação e residual $\left(\mathrm{kg} \mathrm{kg}^{-1}\right)$, respectivamente. $\mathrm{O}$ software utilizado no ajuste do modelo de van Genuchten foi o SWRC, versão 2.00 (Dourado-Neto et al., 2000).

Os parâmetros do modelo de van Genuchten (1980) foram estimados minimizando a soma dos quadrados dos desvios dos valores dos conteúdos de água retidos nas amostras, pelo método iterativo de NewtonRaphson (Dourado Neto et al., 1990). Cada um dos 63 valores (médias das três replicatas) do conteúdo de água retido nas amostras em função das tensões aplicadas foi analisado como bloco, isolando assim os efeitos da sequência de culturas e das camadas de solo, e, portanto, submetidos às 450 combinações. Cada parâmetro do modelo de van Genuchten (1980), para cada uma das 450 combinações, foi comparado ao parâmetro equivalente da curva $\mathrm{C} 1$. Os parâmetros foram submetidos à análise de variância (teste F) e suas médias comparadas pelo teste de Scott-knott a $5 \%$. Para validar os resultados obtidos no presente estudo, ou seja, a eficiência relativa das CRAs, com menor número de tensões, comparativamente à curva de referência (C1), utilizou-se de outro conjunto de dados (outras 189 amostras), referentes a outro tratamento do experimento de longa duração, o qual é constituído pelo cultivo de milho em monocultura como cultura de verão e, na entressafra (cultivo de outono/ inverno), pelo cultivo de milho, girassol, nabo forrageiro, milheto, guandu, sorgo e crotalária.

\section{RESULTADOS E DISCUSSÃO}

Verificou-se considerável variação nos teores de argila do solo (379 a $720 \mathrm{~g} \mathrm{~kg}^{-1}$ de argila) na área experimental (Quadro 1), o que proporcionou ajuste dos dados ao modelo matemático, as curvas de retenção de água no solo (CRA), a partir de dados obtidos sob 
ampla variação textural, o que torna os resultados mais representativos. Como já observado em outros trabalhos (Hodnett \& Tomasella, 2002; Silva et al., 2008), o que corrobora os resultados do presente estudo, observou-se que, quanto maior o teor de argila, maior foi o conteúdo de água retido no solo, para as diferentes tensões (Figura 1). Silva et al. (2008) salientaram a forte influência do teor de argila na retenção de água no solo, em trabalho realizado com Latossolo Vermelho distrófico e com Latossolo Vermelho eutroférrico. Nesse sentido, a figura 1 permite verificar a dependência do conteúdo de água no solo em relação ao teor de argila.

Verificou-se que as combinações de valores de tensões e o número de tensões utilizadas influenciaram no ajuste dos dados ao modelo matemático de van Genuchten (Quadros 3 e 4). Somente um número reduzido de combinações de tensões (Quadro 4) fez com que todos os parâmetros do modelo de van Genuchten $\left(\alpha, \mathrm{n}, \mathrm{m}\right.$ e $\left.U g_{\text {res }}\right)$ apresentassem valores estatisticamente iguais $(\mathrm{p}<0,01)$ àqueles da curva $\mathrm{C} 1$, considerada como referência (Quadro 3). O critério de seleção utilizado para escolha das curvas estatisticamente iguais à $\mathrm{C} 1$ foi o de que todos os valores dos parâmetros do modelo de van Genuchten ( $\alpha, \mathrm{m}, \mathrm{n}$ e $U g_{\text {res }}$ ) fossem estatisticamente iguais, pelo teste de Scott-Knott $(p<0,05)$. Desse modo, as CRAs que apresentaram pelo menos um dos parâmetros do modelo de van Genuchten com valor diferente do seu respectivo na curva $\mathrm{C} 1$ foram consideradas estatisticamente diferentes de $\mathrm{C} 1$ (Quadro 3).

Verificou-se ainda, neste estudo, que, entre as 450 combinações de tensões propostas, aquelas que apresentaram valores de $\alpha, \mathrm{m}, \mathrm{n}$ e $U g_{\text {res }}$ estatisticamente diferentes daqueles da C1 (Quadro 3) não possuíam na combinação de valores de tensões propostas a tensão de $15.000 \mathrm{hPa}$ (Quadro 4). Mesmo quando foi uti-

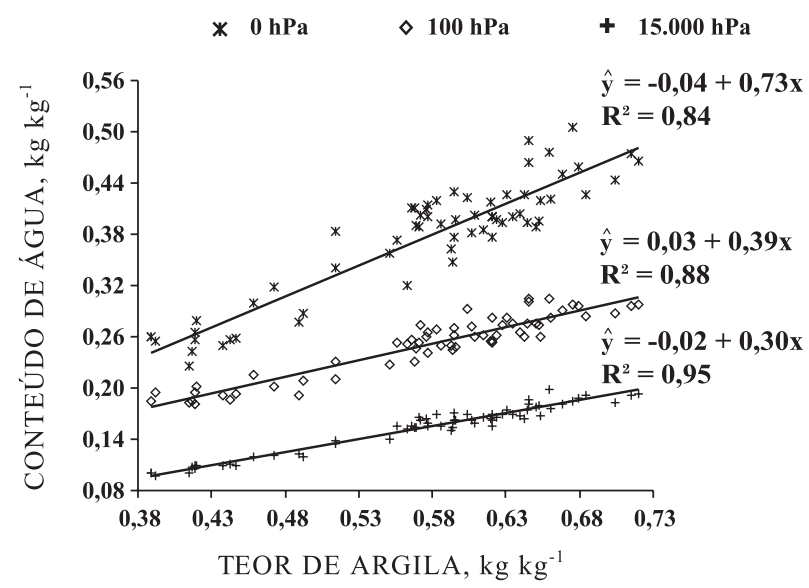

Figura 1. Relação entre o teor de argila do solo e o conteúdo de água retida no solo Latossolo Vermelho eutrófico típico nas tensões de 0,100 e $15.000 \mathrm{hPa}$. lizada uma combinação com elevado número de tensões (12 tensões), esse fato ficou confirmado no presente estudo, o que explica a diferença observada entre as curvas na figura $2 \mathrm{c}$, principalmente a partir da tensão de $200 \mathrm{hPa}$. No presente estudo, esse fato implica afirmar que o modelo não estimou adequadamente o conteúdo de água retido do solo na tensão de $15.000 \mathrm{hPa}$ e que, ao se utilizar o modelo de van Genuchten para elaboração de uma curva característica de retenção de água do solo, deve-se incluir esta tensão no conjunto de tensões utilizadas para isso. Caso contrário, é provável que se obtenham resultados incorretos de avaliação da retenção de água pelo solo e, consequentemente, da disponibilidade de água para as plantas, assim como incertezas na determinação de indicadores da qualidade física do solo derivados da CRA, como o índice S proposto por Dexter (2004).

As combinações com os menores números de tensões e que proporcionaram valores dos parâmetros do modelo de van Genuchten $\left(\alpha, \mathrm{m}, \mathrm{n}\right.$ e $\left.U g_{\text {res }}\right)$ estatisticamente iguais àqueles da curva de referência C1 foram: 0, 80, 300, 700 e $15.000 \mathrm{hPa}$ e 0, 100, 300, 1.000 e $15.000 \mathrm{hPa}$ (Quadro 3). Ou seja, as CRAs, definidas com uma dessas combinações de tensões, sendo os dados ajustados ao modelo de van Genuchten, possibilitaram, portanto, estimativas dos conteúdos de água retidos no solo em diferentes tensões estatisticamente iguais àquelas encontradas quando se utilizou a curva de referência $\mathrm{C} 1$, com um conjunto de 15 valores de tensão, sendo eles: $0,10,20,40,60$, $80,100,200,300,500,700,1.000,3.000,5.000$ e $15.000 \mathrm{hPa}$ (Figura 2a).

Entretanto, no presente estudo, no qual cada amostra, com dimensões de $0,05 \mathrm{~m}$ de diâmetro e $0,05 \mathrm{~m}$ de altura, foi submetida sucessivamente a todas as tensões propostas em cada combinação, o tempo gasto para elaboração de $\mathrm{C} 1$ foi de, aproximadamente, 150 dias. Já para elaboração das CRAs, utilizando-se qualquer um dos seguintes conjuntos de tensões $(0,80,300$, 700 e 15.000 hPa; e 0, 100, 300, 1.000 e 15.000 hPa), foram gastos aproximadamente 30 dias, ou seja, um tempo cinco vezes menor do que o observado na elaboração da curva $\mathrm{C} 1$.

Verificou-se, ainda, que as combinações com as tensões de $0,60,700$ e $15.000 \mathrm{hPa} ; 0,80,700$ e $15.000 \mathrm{hPa}$; e 0, 100, 1.000 e $15.000 \mathrm{hPa}$, todas com quatro valores de tensões, e a combinação $0,20,60$, 100,700 e $15.000 \mathrm{hPa}$, com seis tensões, quando os dados de cada combinação foram ajustados ao modelo de van Genuchten, proporcionaram CRAs que apresentaram um dos parâmetros do modelo $(\alpha, \mathrm{m}, \mathrm{n}$ e $U g_{\text {res }}$ ) estatisticamente diferente do seu respectivo na curva de referência C1 (Quadro 3). Entretanto, essas CRAs proporcionaram estimativas dos conteúdos de água retidos no solo em diferentes tensões estatisticamente iguais às encontradas quando se utilizou a curva de referência C1 (Quadro 5 e Figura 2b,d). Utilizando-se qualquer uma dessas 
Quadro 3. Valores médios dos parâmetros do modelo de van Genuchten (1980) usados na determinação das curvas de retenção de água no solo, levando-se em conta diferentes combinações e números de tensões

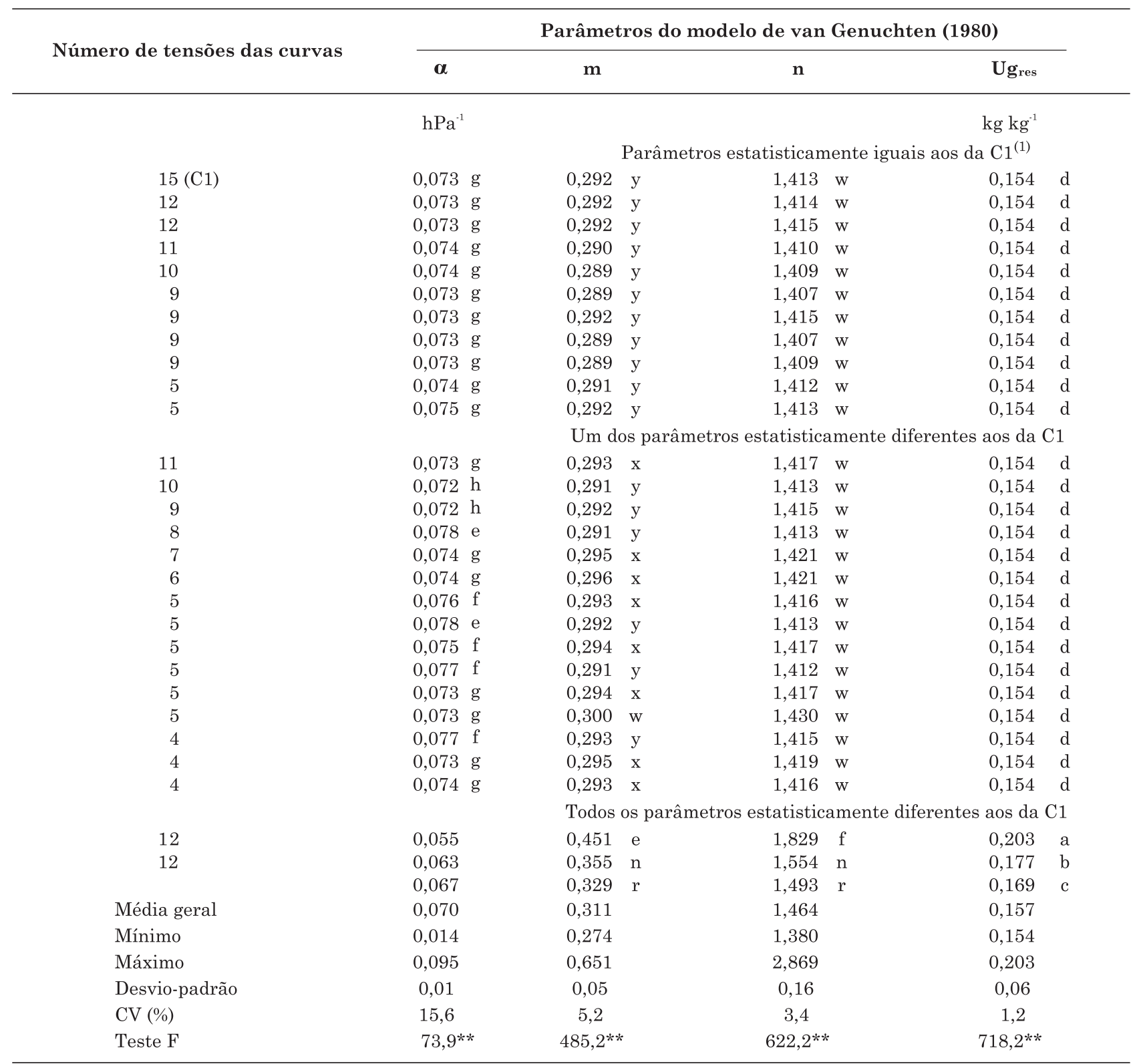

(1) $\mathrm{C} 1$ : curva de referência, ajustada a partir de 15 valores de tensão. Médias seguidas de mesma letra na coluna fazem parte do mesmo grupo estatístico (Scott-knott $\mathrm{p}>0,05)$. ${ }^{* *}$ significativo a $1 \%$.

combinações, o tempo necessário para elaboração da CRA foi de, aproximadamente, 7 a 10 dias menor, quando comparado ao tempo necessário para elaborar as CRAs utilizando as combinações 0, 80, 300, 700 e $15.000 \mathrm{hPa}$ ou $0,100,300,1.000$ e $15.000 \mathrm{hPa}$, as quais apresentaram todos os valores dos parâmetros do modelo de van Genuchten ( $\alpha, \mathrm{m}, \mathrm{n}$ e $\left.U g_{\text {res }}\right)$ estatisticamente iguais aos da curva de referência $\mathrm{C} 1$. $\mathrm{O}$ menor tempo deve-se à substituição de tensões de menores valores $(\leq 100 \mathrm{hPa})$, em detrimento de tensões de maiores valores $(>100 \mathrm{hPa})$, por ocasião da escolha da combinação delas. Para as tensões de menores valores, o equilíbrio hídrico das amostras é atingido em menor tempo, ou seja, aproximadamente um dia para cada tensão em mesa de tensão de areia (Lima \& Silva, 2008). Portanto, a diminuição do tempo e dos custos para a determinação da parte inicial da CRA possibilita a otimização do processo.

No entanto, para determinação do conteúdo de água retido entre as tensões de 100 e 1.000 hPa é necessário usar o aparelho extrator de Richards, o que 
Quadro 4. Combinações de tensões utilizadas no modelo de van Genuchten (1980), as quais geraram os parâmetros $\alpha, \mathrm{m}, \mathrm{n}$ e $\mathrm{Ug}_{\text {res }}$ apresentados do quadro 3

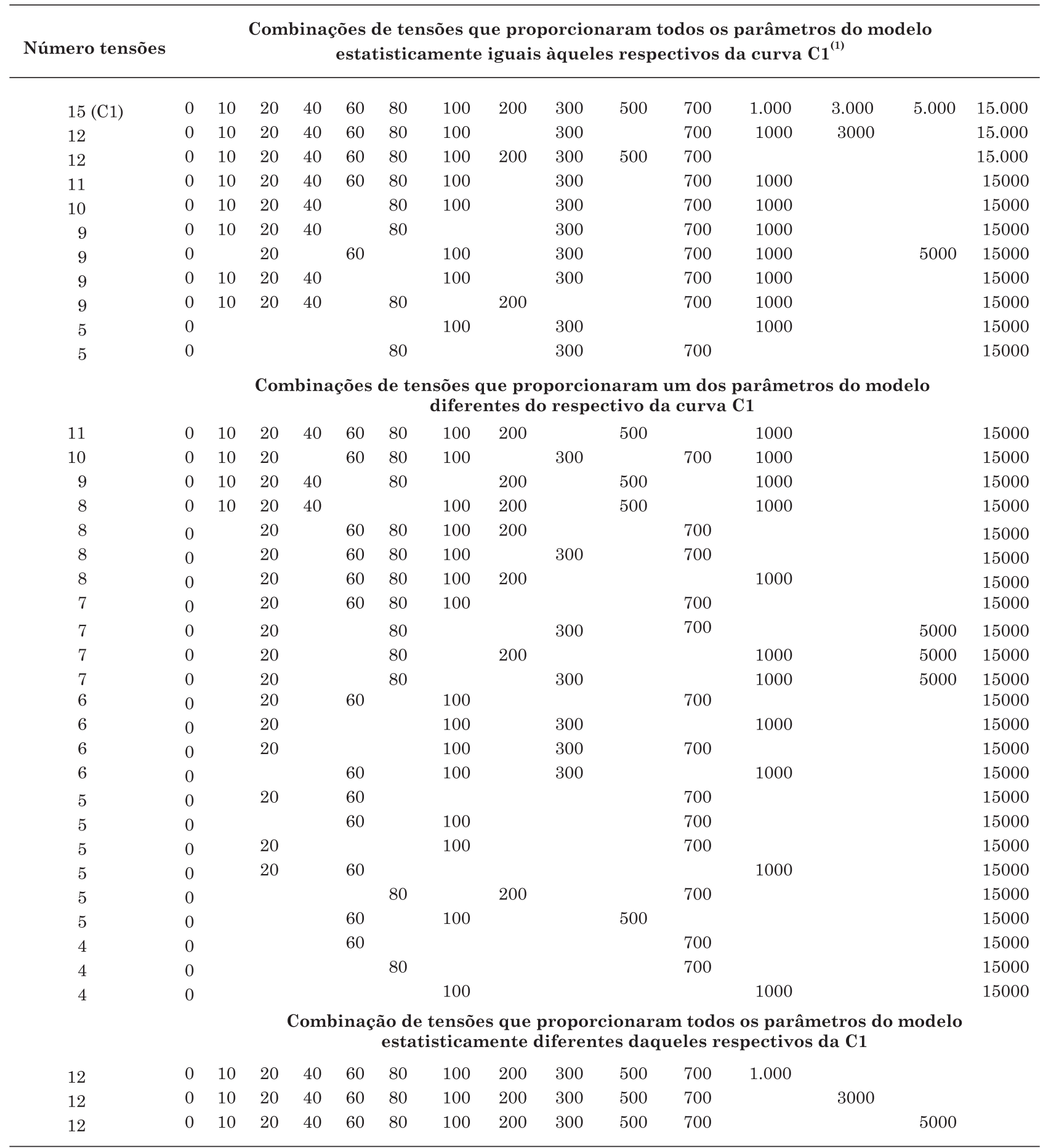

(1) $\mathrm{C} 1$ : curva de referência, ajustada a partir de 15 valores de tensão.

torna a determinação da CRA um processo mais demorado quando se utiliza maior número de tensões de maior valor. Para esse intervalo de tensões, o equilíbrio hídrico das amostras indeformadas de solo (anéis de altura e diâmetro de 0,05 m) pode demorar até 30 dias para ser atingido em cada tensão, dependendo da textura do solo. No presente estudo, o tempo gasto para que o equilíbrio hídrico das amostras fosse atingido foi de aproximadamente 20 dias para cada tensão. 

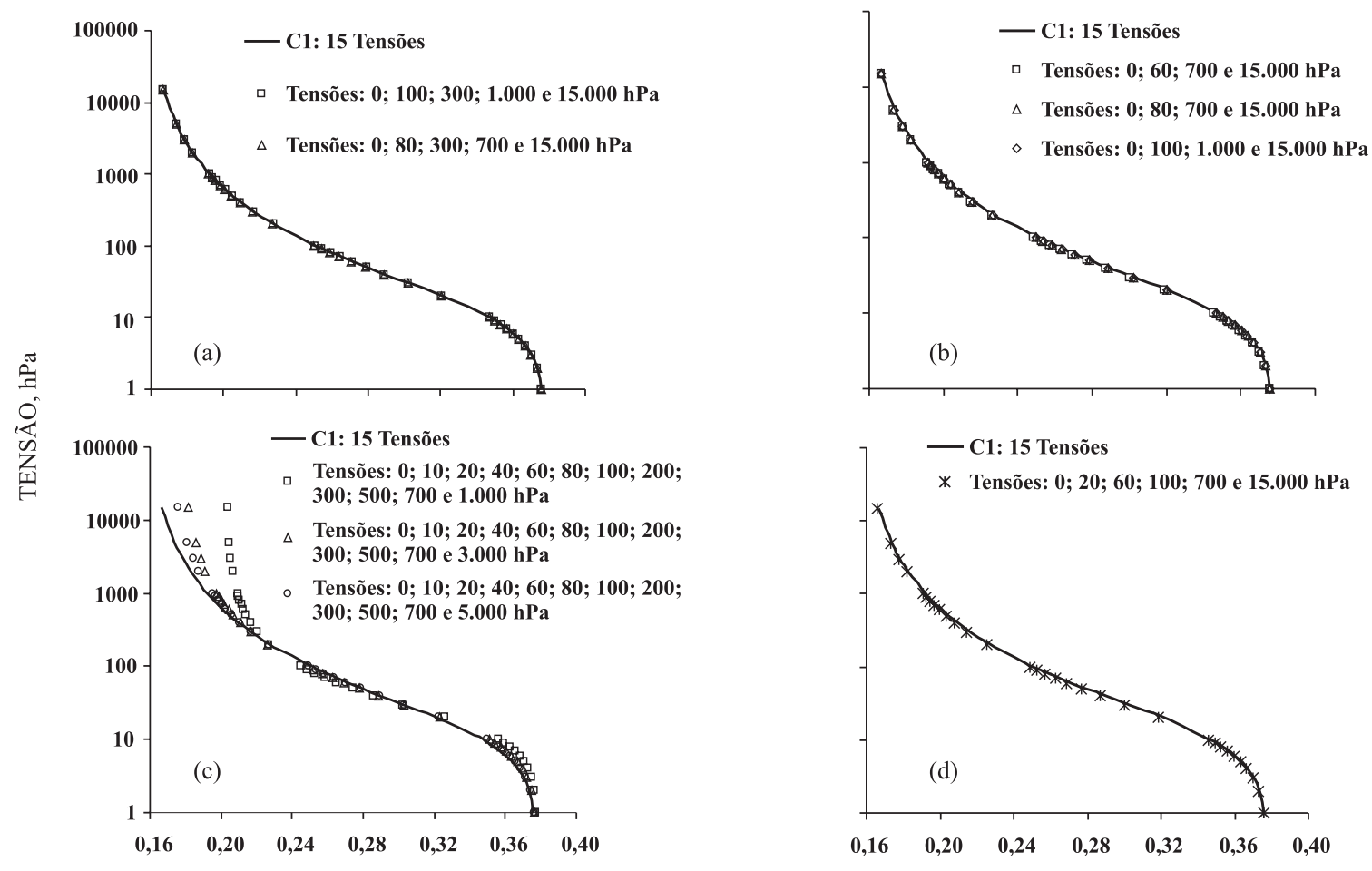

CONTEÚDO DE ÁGUA NO SOLO, $\mathrm{kg} \mathrm{kg}^{-1}$

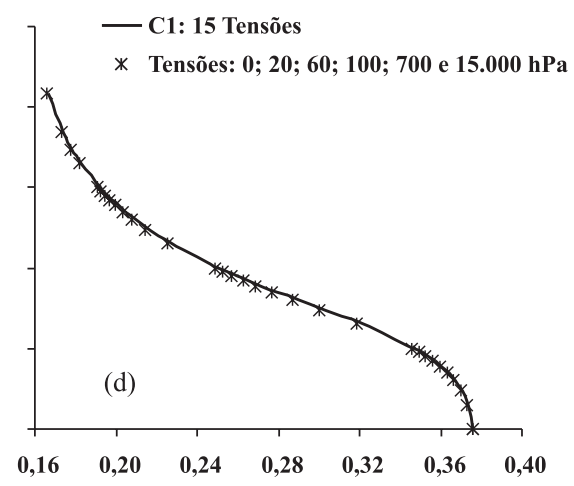

Figura 2. Curvas de retenção de água no solo com: (a) todos os parâmetros iguais aos da curva C1; (b e d) com pelo menos um dos parâmetros diferentes dos de C1; e (c) com todos os parâmetros diferentes dos de C1, ajustados pelo modelo de van Genuchten (1980).

Quadro 5. Conteúdos de água retidos no solo $\left(\mathrm{kg} \mathrm{kg}^{-1}\right)$ estimados pelo modelo de van Genuchten (1980) para diferentes conjuntos de tensões

\begin{tabular}{|c|c|c|c|c|c|c|c|c|}
\hline \multirow{2}{*}{ Número de tensões das curvas } & \multicolumn{8}{|c|}{ Tensão (hPa) } \\
\hline & 10 & 20 & 60 & 100 & 200 & 300 & 1.000 & 15.000 \\
\hline & \multicolumn{8}{|c|}{ Conteúdo de água no solo $\left(\mathrm{kg} \mathrm{kg}^{-1}\right)$} \\
\hline $15(\mathrm{C} 1)^{(1)}$ & 0,35 & 0,32 & 0,27 & 0,25 & 0,23 & 0,22 & 0,19 & 0,17 \\
\hline $6^{(2)}$ & 0,35 & 0,32 & 0,27 & 0,25 & 0,23 & 0,22 & 0,19 & 0,17 \\
\hline $5^{(3)}$ & 0,35 & 0,32 & 0,27 & 0,25 & 0,23 & 0,22 & 0,19 & 0,17 \\
\hline $4^{(4)}$ & 0,35 & 0,32 & 0,27 & 0,25 & 0,23 & 0,22 & 0,19 & 0,17 \\
\hline $\mathrm{CV}(\%)^{(5)}$ & 1,2 & 1,5 & 1,4 & 1,3 & 1,3 & 1,2 & 1,1 & 1,1 \\
\hline
\end{tabular}

(1) Curva C1 (tensões: 0, 10, 20, 40, 60, 80, 100, 200, 300, 500, 700, 1.000, 3.000, 5.000 e 15.000 hPa). ${ }^{(2)}$ (tensões: 0, 20, 60, 100, 700 e $15.000 \mathrm{hPa}$ ). ${ }^{(3)}$ (tensões: 0, 100, 300, 1.000 e $15.000 \mathrm{hPa}$; ou 0, 80, 300, 700 e $15.000 \mathrm{hPa}$ ). ${ }^{(4)}$ (tensões: 0, 60, $700 \mathrm{e} 15.000 \mathrm{hPa}$; 80,700 e $15.000 \mathrm{hPa}$; 0, 100, 1.000 e $15.000 \mathrm{hPa}$ ).

Apesar de ser considerada tensão de alto valor, o equilíbrio hídrico das amostras de solo submetidas à tensão de $15.000 \mathrm{hPa}$ na câmara de Richards foi atingido, no presente estudo, em tempo relativamente curto, ou seja, de aproximadamente três dias. Isso foi possível porque utilizaram-se amostras indeformadas de solo com 0,01 $\mathrm{m}$ de altura. Para tensões com valores maiores que $1.000 \mathrm{hPa}$, podem-se utilizar amostras deformadas de terra com menor altura, as quais são acondicionadas em anéis de borracha ou PVC e submetidas às tensões diretamente sobre a placa porosa, utilizada na câmara de Richards (Embrapa, 1997). Dessa maneira, as amostras atingem o equilíbrio hídrico em menor tempo, quando comparado ao tempo, relativamente maior (aproximadamente 20 dias), para a determinação do conteúdo de água em amostras indeformadas de solo acondicionadas em anéis volumétricos com altura de 0,05 m. Contudo, 
esse procedimento é recomendado para determinação de conteúdos de água em amostras de solo nas tensões maiores que $1.000 \mathrm{hPa}$ (Embrapa, 1997), pois a retenção de água nesses casos tem maior relação com o teor de argila do solo do que com sua estrutura (Hodnett \& Tomasella, 2002; Silva et al., 2008).

Na literatura, são escassas as informações sobre quais tensões devem ser usadas na determinação da CRA. Silva et al. (2006), ao avaliarem quais e quantas tensões devem ser levadas em conta na determinação da CRA de solos sob Cerrado, utilizando centrífuga, recomendaram oito tensões: 10, 30, 60, 100, 350, 840, 6.110 e $15.150 \mathrm{hPa}$. Esses autores afirmaram que curvas com menos de oito tensões não devem ser utilizadas.

A Embrapa (1997) recomenda, para determinação da CRA obtida com aparelho de extrator de Richards, as seguintes tensões: 100, 330, 1.000, 5.000 e $15.000 \mathrm{hPa}$. No presente estudo, verificou-se que o tempo gasto para determinar as CRAs, utilizando qualquer um dos seguintes conjuntos de tensões: (0, 80, 300, 700 e $15.000 \mathrm{hPa}),(0,100,300,1.000$ e $15.000 \mathrm{hPa}),(0,20,60,100,700$ e $15.000 \mathrm{hPa})$, foi menor que o tempo necessário para a determinação da CRA utilizando o conjunto de tensões recomendado e com a mesma acurácia que C1.

A fim de validar os resultados obtidos no presente estudo, ou seja, a eficiência relativa das CRAs, com menor número de tensões e mesma acurácia, quando comparadas com a curva de referência (C1), utilizouse novo conjunto de dados (outras 189 amostras), referente a outro tratamento do experimento de longa duração, o qual é constituído por diferente sequência de culturas de verão e inverno. Assim, valores dos conteúdos de água retidos no solo em diferentes tensões, provenientes de amostras do solo de outro tratamento, foram ajustados ao modelo de van Genuchten, utilizando-se os seguintes conjuntos de tensões: $(0,80,300,700$ e $15.000 \mathrm{hPa}),(0,100,300$, 1.000 e $15.000 \mathrm{hPa}),(0,20,60,100,700$ e $15.000 \mathrm{hPa})$, $(0,60,700$ e $15.000 \mathrm{hPa}),(0,80,700$ e $15.000 \mathrm{hPa}) \mathrm{e}$ $(0,100,1.000$ e $15.000 \mathrm{hPa})$, os quais se mostraram, inicialmente, eficientes para estimar o conteúdo de água retido no solo, quando comparados aos da curva de referência $\mathrm{C} 1$, porém com menor número de tensões e em menor período de tempo.

Verificaram-se diferentes valores dos parâmetros $m$ e n do modelo de van Genuchten, quando comparados àqueles da curva de referência $\mathrm{C} 1$, para as combinações com quatro valores de tensões $(0,60,700$ e $15.000 \mathrm{hPa} ; 0,80,700$ e $15.000 \mathrm{hPa}$; e 0, 100, 1.000 e $15.000 \mathrm{hPa})$ e com seis valores $(0,20,60,100,700 \mathrm{e}$ $15.000 \mathrm{hPa})$. Para as combinações $(0,80,300,700$, $15.000 \mathrm{hPa})$ e $(0,100,300,1.000$ e $15.000 \mathrm{hPa})$, os valores dos parâmetros de van Genuchten foram iguais aos respectivos da curva C1 (Quadro 6).

Quando se observam os dados apresentados no quadro 7, entretanto, verifica-se que esses conjuntos de tensões proporcionaram estimativas dos conteúdos de água retidos no solo estatisticamente iguais às encontradas quando se utilizou a curva de referência C1. Esse fato demonstra, portanto, que, mesmo

Quadro 6. Valores dos parâmetros do modelo de van Genuchten (1980) gerados a partir do novo conjunto de dados, provenientes de amostras coletadas em outro tratamento, e utilizados para validação das curvas de retenção de água do solo

\begin{tabular}{ccccc}
\hline $\begin{array}{c}\text { Número de tensões } \\
\text { das curvas }\end{array}$ & $\boldsymbol{\alpha}$ & $\mathbf{m}$ & $\mathbf{n}$ & $\boldsymbol{U g}_{\text {res }}$ \\
\hline & $\mathrm{hPa}^{-1}$ & & & $\mathrm{~kg} \mathrm{~kg}^{-1}$ \\
$15(\mathrm{C} 1)^{(1)}$ & 0,081 & $0,283 \mathrm{~b}$ & $1,396 \mathrm{~b}$ & 0,148 \\
$6^{(2)}$ & 0,085 & $0,288 \mathrm{a}$ & $1,405 \mathrm{a}$ & 0,148 \\
$5^{(3)}$ & 0,083 & $0,283 \mathrm{~b}$ & $1,395 \mathrm{~b}$ & 0,148 \\
$4^{(4)}$ & 0,082 & $0,288 \mathrm{a}$ & $1,405 \mathrm{a}$ & 0,148 \\
$\mathrm{CV}(\%)$ & 13,0 & 2,9 & 1,2 & 0,1 \\
Teste F & $1,7^{\mathrm{ns}}$ & $9,7^{* *}$ & $9,18^{* *}$ & $0,1^{\mathrm{ns}}$ \\
\hline
\end{tabular}

(1) Curva C1 ( tensões: 0, 10, 20, 40, 60, 80, 100, 200, 300, 500, 700, 1.000, 3.000, 5.000 e $15.000 \mathrm{hPa})$. ${ }^{(2)}$ (tensões: 0, 20, 60, 100, 700 e $15.000 \mathrm{hPa}$ ). ${ }^{(3)}$ (tensões: 0, 100, 300, $1.000 \mathrm{e}$ $15.000 \mathrm{hPa}$; ou $0,80,300,700$ e $15.000 \mathrm{hPa}$ ). ${ }^{(4)}$ (tensões: 0,60 , 700 e $15.000 \mathrm{hPa}$; 0, 80, 700 e $15.000 \mathrm{hPa}$; 0, 100, 1.000 e $15.000 \mathrm{hPa})$.

Quadro 7. Conteúdos de água retidos no solo $\left(\mathrm{kg} \mathrm{kg}^{-1}\right)$ em diferentes tensões, estimados pelas curvas de retenção de água do solo, quando se utilizaram os parâmetros do modelo de van Genuchten gerados a partir do novo conjunto de dados, provenientes de amostras coletadas em outro tratamento

\begin{tabular}{|c|c|c|c|c|c|c|c|c|}
\hline \multirow{2}{*}{ Número de tensões das curvas } & \multicolumn{8}{|c|}{ Tensão (hPa) } \\
\hline & 10 & 20 & 60 & 100 & 200 & 300 & 1.000 & 15.000 \\
\hline & \multicolumn{8}{|c|}{ Conteúdo de água no solo $\left(\mathrm{kg} \mathrm{kg}^{-1}\right)$} \\
\hline $15(\mathrm{C} 1)^{(1)}$ & 0,34 & 0,31 & 0,26 & 0,25 & 0,22 & 0,21 & 0,19 & 0,16 \\
\hline $6^{(2)}$ & 0,33 & 0,31 & 0,26 & 0,24 & 0,22 & 0,21 & 0,19 & 0,16 \\
\hline $5^{(3)}$ & 0,34 & 0,31 & 0,26 & 0,25 & 0,22 & 0,21 & 0,19 & 0,16 \\
\hline $4^{(4)}$ & 0,34 & 0,31 & 0,26 & 0,24 & 0,22 & 0,21 & 0,19 & 0,16 \\
\hline CV (\%) & 1,0 & 1,2 & 0,8 & 0,6 & 0,4 & 0,4 & 0,6 & 0,6 \\
\hline
\end{tabular}

${ }^{(1)}$ Curva C1 (tensões: 0, 10, 20, 40, 60, 80, 100, 200, 300, 500, 700, 1.000, 3.000, 5.000 e 15.000 hPa). ${ }^{(2)}$ (tensões: 0, 20, 60, 100, 700 e $15.000 \mathrm{hPa}){ }^{\left({ }^{(3)}\right.}$ (tensões: 0, 100, 300, 1.000 e $15.000 \mathrm{hPa}$; ou 0, 80, 300, 700 e $\left.15.000 \mathrm{hPa}\right)$. ${ }^{(4)}$ (tensões: $0,60,700$ e $15.000 \mathrm{hPa}$; 0 , $80,700$ e $15.000 \mathrm{hPa} ; 0,100,1.000$ e $15.000 \mathrm{hPa})$. 
utilizando-se outro conjunto de dados, os resultados foram semelhantes aos observados com o conjunto de dados inicial, o que valida os resultados encontrados no presente estudo.

Esse fato implica afirmar que é possível estimar o conteúdo de água retido no solo utilizando-se uma curva característica de retenção de água do solo com menor número de tensões, em menor período de tempo e com acurácia adequada. Contudo, é essencial definir quais tensões serão utilizadas no modelo matemático de van Genuchten, para que proporcionem resultados e custos desejados.

\section{CONCLUSÕES}

1. A hipótese deste estudo foi confirmada, ou seja, é possível determinar a CRA com menor número de tensões e reduzindo seu tempo de obtenção, sem, contudo, comprometer a acurácia dos resultados.

2. A tensão de $15.000 \mathrm{hPa}$ deve estar contida na combinação de tensões a serem utilizadas para determinação da curva característica de retenção de água quando se utiliza o modelo matemático de van Genuchten para ajuste dos dados.

3. As curvas características de retenção de água do solo, definidas com as combinações de tensões ( 0 , 60, 700 e $15.000 \mathrm{hPa}),(0,80,700$ e $15.000 \mathrm{hPa}),(0$, $100,1.000$ e $15.000 \mathrm{hPa}),(0,20,60,100,700$ e $15.000 \mathrm{hPa}),(0,80,300,700$ e $15.000 \mathrm{hPa})$ e $(0,100$, $300,1.000$ e $15.000 \mathrm{hPa}$ ), sendo os dados ajustados ao modelo de van Genuchten, proporcionaram estimativas dos conteúdos de água retidos em diferentes tensões no Latossolo Vermelho eutrófico, sob sistema de semeadura direta, com a mesma acurácia, quando comparada à curva de referência, partindo-se de um conjunto de 15 valores de tensões.

\section{AGRADECIMENTOS}

Os autores agradecem à FAPESP (Fundação de Amparo à Pesquisa do Estado de São Paulo), pelo auxílio financeiro ao projeto, e à CAPES (Coordenação de Aperfeiçoamento de Pessoal de Nível Superior), pela bolsa concedida ao primeiro autor.

\section{LITERATURA CITADA}

BLAINSKI, E.; TORMENA, C.A.; FIDALSKI, J. \& GUIMARAES, R.M.L. Quantificação da degradação física do solo por meio da curva de resistência do solo à penetração. R. Bras. Ci. Solo, 32:975-983, 2008.

DEXTER, A.R. Soil physical quality - Part I. Theory, effects of soil texture, density, and organic matter, and effects on root growth. Geoderma, 120:201-214, 2004.

DEXTER, A.R.; CZYZ, E.A. \& GAPE. O.P. A method for prediction of soil penetration resistance. Soil Till. Res., 93:412-419, 2007.
DEXTER, A.R.; CZYZ, E.A.; RICHARD, G. \& RESZKOWSKA, A. A user-friendly water retention function that takes account of the textural and structural pore spaces in soil. Geoderma, 143:243-253, 2008.

DOURADO-NETO, D.; JONG VAN LIER, Q.; BOTREL, T.A. \& LIBARDI, P.L. Programa para confecção da curva de retenção de água no solo utilizando o modelo de Genuchten. Eng. Rural, 1:92-102, 1990.

DOURADO-NETO, D.; NIELSEN, D.R.; HOPMANS, J.W.; REICHARDT, K. \& BACCHI, O.O.S. Software to model soil water retention curves (SWRC, version 2.00). Sci. Agric., 57:191-192, 2000.

EMPRESA BRASILEIRA DE PESQUISA AGROPECUÁRIA EMBRAPA. Centro Nacional de Pesquisa de Solos. Sistema Brasileiro de Classificação de Solos. Brasília, 2006. 412p.

EMPRESA BRASILEIRA DE PESQUISA AGROPECUÁRIA EMBRAPA. Centro Nacional de Pesquisa de Solos. Manual de métodos de análise de solo. 2.ed. Rio de Janeiro, 1997. $212 p$

GARDNER, W.H. Water content. In: KLUTE, A., ed. Methods of soil analysis: Physical and mineralogical methods. 2.ed. Madison, American Society of Agronomy, 1986. Part 1. p.493-544.

HODNETT, M.G. \& TOMASELLA, J. Marked differences between van Genuchten soil water-retention parameters for temperate and tropical soils: A new water-retention pedo-transfer function developed for tropical soils. Geoderma, 108:155-180, 2002.

KLUTE, A. Water retention: Laboratory methods. In: KLUTE, A., ed. Methods of soil analysis: Physical and mineralogical methods. 2.ed. Madison, American Society of Agronomy, 1986. Part 1. p.635-662.

LIMA, H.V. \& SILVA, A.P. Mesa de tensão com areia: Procedimentos para montagem e validação. R. Bras. Ci. Solo, 32:2209-2214, 2008.

MACHADO, J.L.; TORMENA, C.A.; FIDALSKI, J. \& SCAPIM, C.A. Inter-relações entre as propriedades físicas e os coeficientes da curva de retenção de água de um Latossolo sob diferentes sistemas de uso. R. Bras. Ci. Solo, 32:495502,2008 .

MORAES, S.O.; LIBARDI, P.L. \& DOURADO NETO, D. Problemas metodológicos na obtenção da curva de retenção. Sci. Agric., 50:383-392, 1993.

SILVA, A.P.; TORMENA, C.A.; FIDALSKI, J. \& IMHOFF, S. Funções de pedotransferência para as curvas de retenção de água e de resistência do solo à penetração. R. Bras. Ci. Solo, 32:1-10, 2008.

SILVA, E.M.; LIMA, J.E.F.W.; AZEVEDO, J.A. \& RODRIGUES, L.N. Valores de tensão na determinação da curva de retenção de água de solos do Cerrado. Pesq. Agropec. Bras., 41:323-330, 2006.

TORMENA, C.A.; SILVA, A.P.; IMHOFF, S.D.C. \& DEXTER, A.R. Quantification of the soil physical quality of a tropical oxisol using the S index. Sci. Agric., 65:56-60, 2008.

van GENUCHTEN, M.T.A. A closed-form equation for predicting the hydraulic conductivity of unsaturated soils. Soil Sci. Soc. Am. J., 44:892-897, 1980. 